\title{
Relative cue properties of novel-tasting substances in avoidance conditioning*
}

\author{
JOHN F. WING and LARRY A. BIRCH $\dagger$ \\ Behavior Research Laboratory, Antioch College, Yellow'Springs, Ohio 45387
}

\begin{abstract}
Rats injected with lithium chloride on a day when they tasted a novel substance, saccharin, subsequently showed avoidance, or bait shyness. However, magnitude of avoidance depended upon the substances given the rats on the days just before and after injection day. Maximum avoidance was found for rats given a familiar, contrasting substance (water) on the days before and after, whereas minimum avoidance was shown by rats given saccharin on those days (as well as on injection day). Intermediate avoidance was found for rats given water on either the day before or the day after. Results confirm the importance of contrasting taste cues in successive discrimination tasks employed in bait-shyness studies.
\end{abstract}

Garcia, Ervin, and Koelling (1967) proposed a toxicity test based upon the bait-shyness phenomenon, i.e., the ability of an animal to associate subsequent malaise with the taste of a food it has sampled. Their test consists of conditional pairings of a novel-tasting substance like saccharin and injections of a toxin. Degree of subsequent avoidance of the food substance is used as a measure of the severity of the toxic effects experienced by the animal. The usual experimental design involves conditional pairings of the novel substance with toxic injections every other day, while on intervening days the animal receives a familiar substance with saline injections (Garcia et al, 1966, 1968). Such a design is a successive discrimination task in which the animal has the opportunity to compare the outcome of each day's taste experience in terms of its toxic vs nontoxic effects. Although this design produces effective conditioning, very little is known about the reasons for its effectiveness. Is it essential, for example, that contrasting tastes with differential outcomes be presented on alternate days, or is the presentation of a novel-tasting substance followed by toxin-induced illness sufficient to produce avoidance of that substance? Revusky and Bedarf (1967) have argued that absolute novelty is an optimal as well as sufficient condition for establishing an aversion. They suggest that investigatory reflexes aroused by novel foods may predispose rats to associate illness with them, so that given both a novel and a familiar substance an animal will associate a subsequent illness with the novel substance. This proposition assigns a relatively minor role to the differential cues and outcomes normally considered essential to discrimination learning. However, in the present study, we have found that the sequence of taste experiences given to an animal (and their differential outcomes) is an important determiner of the magnitude of avoidance conditioning.

\footnotetext{
*This research was supported by the Biochemical Research Laboratory, Dow Chemical Company, Midland, Michigan.

tBoth authors are presently in the Department of Psychology at Wittenberg University, Spring field. Ohio 45501.
}

We investigated magnitude of an avoidance response conditioned to a relatively novel substance (saccharin) as a function of the positioning of "water days" (a familiar substance) before and/or after the toxin-injection day. The design consisted of presenting the familiar substance of water to each of four groups: water before and after (WBA), water before only (WBO), water after only (WAO), and water neither before nor after (WNBA). Thus, we tried to assess the relative value of a "water-well day" positioned before, after, before and after, or neither before nor after a "saccharin-sickness day."

It was expected that maximum saccharin avoidance would be shown by the WBA experimental group because they had the benefit of a contrasting, familiar substance (water) on the days both preceding and following the saccharin-toxin injection day. In short, they had the best opportunity to form the contrasting association: "saccharin-sickness" vs "water-well." It was also expected that the WNBA experimental group would show the least saccharin avoidance because they lacked both contrasting water days. No prediction was made concerning the relative order of the two remaining experimental groups; however, it was predicted that both would be intermediate to the WBA and the WNBA groups.

\begin{abstract}
METHOD
Subjects and Preexperimental Phase

Sixty male Holtzman rats, 90 days old, were individually housed. Food was alway's available in their cages, but liquid was available only $10 \mathrm{~min}$ daily during the preexperimental phase. The rats were habituated to this drinking schedule for 5 days before the treatments began, and this schedule was continued throughout.
\end{abstract}

\section{Experimental Phase}

On the sixth day. one of the four sequences of saccharin and water days shown in Table 1 was given to each of four experimental groups $(N=10)$ and to their yoked control groups $(N=5)$. Experimental groups were injected with $66 \mathrm{mg} / \mathrm{kg}$ lithium chloride on Day 8 , but received saline injections on all other days of the experiment. Control groups were injected with 
Table 1

Sequence of Substances Given Rats Under the Four Design Treatments

\begin{tabular}{|c|c|c|c|c|c|c|c|}
\hline \multirow{3}{*}{$\begin{array}{c}\text { Design } \\
\text { Treatments }\end{array}$} & \multicolumn{7}{|c|}{ Days of Experiment } \\
\hline & \multirow{2}{*}{$\frac{\text { Habituation }}{1 \ldots 5}$} & \multicolumn{5}{|c|}{ Conditioning } & \multirow{2}{*}{$\frac{\text { Extinction and Test* }}{11 \ldots 15}$} \\
\hline & & 6 & 7 & 8 & 9 & 10 & \\
\hline Before and After & $W \ldots W$ & S & W & $\mathrm{S}$ & w & $S^{*}$ & $S^{*} \ldots S^{*}$ \\
\hline Before Only & $w \ldots w$ & $\mathrm{~S}$ & W & $\mathrm{S}$ & $\mathrm{S}^{*}$ & $\mathrm{~S}^{*}$ & $S^{*} \ldots S^{*}$ \\
\hline After Only & $w \ldots w$ & W & $\mathrm{S}$ & $\mathrm{S}$ & $W$ & $S^{*}$ & $S^{*} \ldots S^{*}$ \\
\hline Neither Before Nor After & $w \ldots w$ & $\mathbf{W}$ & $\mathrm{S}$ & $\mathrm{S}$ & $\mathrm{S}^{*}$ & $S^{*}$ & $S^{*} \ldots S^{*}$ \\
\hline
\end{tabular}

Note-Each treatment was given to an experimen:al group $(N=10)$ and a yoked control group $(N=5)$. The words "before" and "after" refer to whether or not water was offered on Dav' 7 and 9. All groups tasted saccharin (S) on Day 8. Experimental groups were given toxic injections on this day, whereas controls were given saline injections. $S=$ saccharin, $W=$ water.

a saline solution on every day, including Day 8 . Injections were given intraperitoneally within a period of $30 \mathrm{~min}$. beginning immediately after the 10 -min drinking session. Order of injection was counterbalanced across groups. Table 1 shows the unique combination of saccharin days (S) and water days (W) given to each yoked pair of groups during the 3-day period of Days 7, 8 . and 9 . To produce the desired sequences (while giving all groups the same amount of saccharin experience), it was necessary that the groups get 1 saccharin day and 1 water day in different orders on Days 6 and 7. It was also necessary that the first saccharin test day following the injection day be delayed by 1 day in the WBA and WAO groups so that they could experience a water day first. Therefore, their first test after injection came on Day 10 rather than on Day 9.

\section{RESULTS AND DISCUSSION}

Figure 1 shows the magnitude of saccharin avoidance on the first test day. Differences in both means and medians are shown for each experimental group and its yoked control. Both these measures of central tendency agree. As predicted, the maximum difference obtained was for the WBA experimental group which drank about $9 \mathrm{ml}$ less saccharin that its yoked control group. As predicted, the minimum difference was between the WNBA groups, and intermediate differences were obtained for the WBO and WAO groups. Statistical analysis confirms this. An analysis of variance applied to the absolute saccharin intake scores showed a significant toxicity effect $(F=36.33, p<.001)$, a significant design effect due to positioning of water days $(F=4.19$, $\mathrm{p}<.02)$, and a nonsignificant interaction effect $(\mathrm{F}=$ $1.71, \mathrm{p}>.20$ ). Critical difference tests applied to the differences between experimental and control group means showed that all the experimental groups differed significantly $(p<.05$ or better $)$ from their yoked control groups, except the WNBA comparison. Thus, any avoidance conditioning which may have been obtained under this particular condition was not of sufficient magnitude to justify rejection of the null hypothesis. After Day 8, all of those experimental groups which had shown a statistically reliable conditioned avoidance also showed a gradual extinction, and by Day 15 they no longer displayed significantly less saccharin intake than their yoked control groups.

Unfortunately, some of these significant differences between groups (attributed here to positioning of water days) could have been due to other factors, most notably to time-dependent drug effects. Since the first test day for the WBA and WAO groups was a day later than for the other two groups, they might have been at a later stage of recovery from poisoning. What might have been the effects of this difference in time of testing? Nachman (1963) reports that rats poisoned with lithium avoid fluids until $7.8 \mathrm{~h}$ after poisoning, when intense drinking begins which serves to flush toxin from the system (antidotal polydipsia). He has studied the actual time course of fluid intake by both poisoned and nonpoisoned rats. He reports that nonpoisoned rats drink more than poisoned rats during the first $24 \mathrm{~h}$, but that the poisoned rats eventually overtake them in rate of drinking as a result of polydipsia, and the net effect is that they drink more during the second $24 \mathrm{~h}$. However, by the end of $48 \mathrm{~h}$, there are no remaining differences

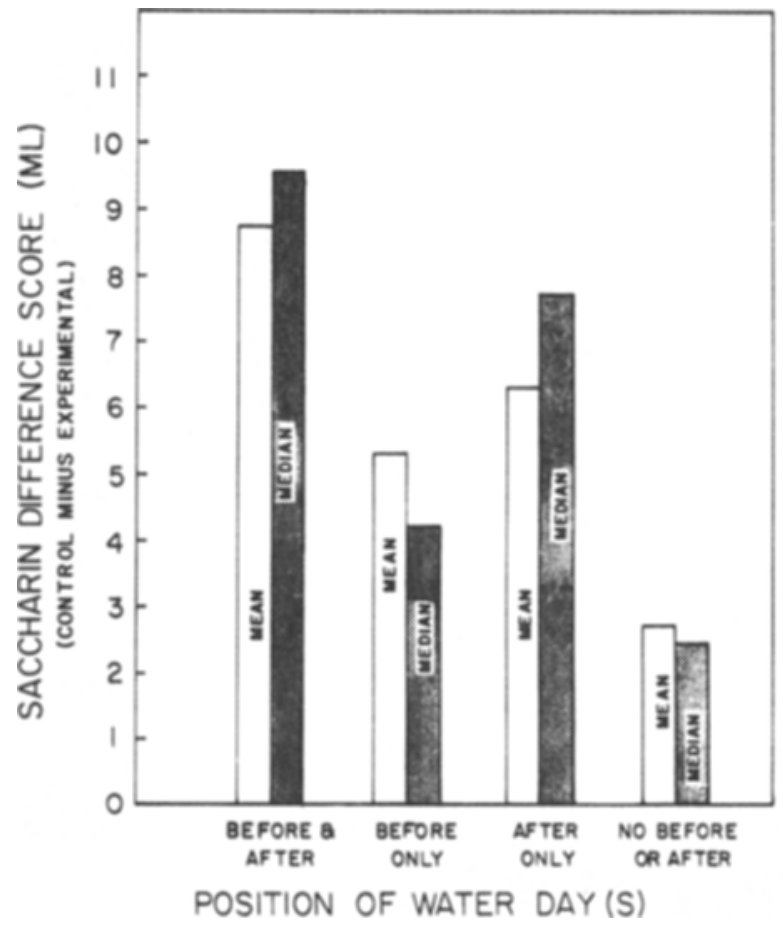

Fig. 1. Effect of positioning of water day(s) on conditioned avoidance of saccharin as measured by the saccharin difference score. 
between poisoned and nonpoisoned rats in rate of drinking, total fluid intake, or body weight.

A simple extension of Nachman's findings would suggest that the WBA and WAO groups (which were both tested $48 \mathrm{~h}$ after poisoning) essentially had recovered from lithium poisoning and would have been drinking at the same rate as their yoked-control groups except for the presence of conditioned aversion. However, the WBO and WNBA groups (which were tested only $24 \mathrm{~h}$ after poisoning) still would be drinking less than their yoked control groups because of antidotal polydipsia and quite apart from any conditioned aversion. This effect would cause artificially inflated C-E difference scores for these two treatment conditions and hence minimize overall differences between treatment effects.

Such a simple extrapolation of Nachman's findings may not be in order, however. In his study, rats had continuous access to fluid for the full $48 \mathrm{~h}$ following lithium poisoning, whereas in the present study, rats were continuously deprived except for the daily $10-\mathrm{min}$ test sessions. It is possible, then, that the antidotal polydipsia (which normally appears at $7.8 \mathrm{~h}$ ) was delayed anywhere up to $24 \mathrm{~h}$ later, at which time the first fluid was available to flush out the poison. If such were the case, all experimental groups in this study would have shown a marked increase in fluid intake $24 \mathrm{~h}$ after poisoning, and this would have decreased the C.E difference scores for the WBO and WNBA groups, which were actually tested at $24 \mathrm{~h}$. Under this delayed polydipsia interpretation, the effect of this artifact would be to maximize overall differences between treatments.

A direct test between nondelayed and delayed polydipsia hypotheses was made by comparing fluid intake for all experimental groups $24 \mathrm{~h}$ after poisoning. Such an analysis showed no evidence of postponed antidotal polydipsia, but did support nonpostponed polydipsia. Comparison of saccharin intake on Days 8 and 9 for WBO and WNBA groups showed decreases of $36.2 \%$ and $13.2 \%$, respectively. These changes were statistically significant for both the WBO group $(\mathrm{t}=$ $6.00, \mathrm{p}<.001)$ and the WNBA group $(\mathrm{t}=8.82$, $\mathrm{p}<.001$ ). Of course, these differences include the main treatment effect of conditioned aversion to saccharin. More importantly, for the WBO and WAO groups, a comparison was made of water intake on Day 9 with their very last water day. It showed decreases of $29.3 \%$ and $13.8 \%$, respectively. Again, the differences between the two sets of scores is statistically significant for both the WBA group $(\mathrm{t}=7.60, \mathrm{p}<.001)$ and the WAO group $(t=3.28, p<.01)$. If these latter groups had been in a marked state of delayed antidotal polydipsia on Day 9 , they certainly would have consumed more water on that day than on the preceding water day. This would be especially so since this was the contrasting substance never directly associated with sickness. Thus, all the evidence points to the presence of a nondelayed antidotal polydipsia, which would have been a process operating against the main hypotheses advanced in this study.

Of course, other temporal factors besides polydipsia could have been operating in a fashion which artifactually produced the results here attributed to contrasting cues. However, we have no idea what these processes might be or how they might have operated in time. Thus, it seems reasonable to conclude that the treatment conditions actually rank order, as shown in Fig. 1. But even if this did not hold, at the very least, the present study has shown greater conditioning within the following pairs of treatments: WBO is greater than WNBA and WBA is greater than WAO. These findings, in effect, demonstrate the importance of giving 1 day with a contrasting substance before the critical day of avoidance conditioning. Whether or not this is a critical operation under all conditions cannot be ascertained from the present study. It might not obtain in studies which employ long delays between CS and UCS. for example. In this study, injections began immediately after ingestion of saccharin, and all rats had been injected within a $30-\mathrm{min}$ period. But within the conditions of this experiment, it has been shown that the effectiveness of a relatively novel substance like saccharin as a cue in avoidance conditioning depends in large measure upon its temporal conditioning vis à vis a contrasting substance like water. In short, to obtain reliable conditioning, the animal must be given maximum opportunities for discriminating the differential outcomes of sampling substances which differ in taste and smell.

\section{REFERENCES}

Garcia, J.. Ervin, F. A.. \& Koelling, R. A. Learning with prolonged delay of reinforcement. Psychonomic Science. $1966,5,121$.

Garcia, J., Ervin, F. R., \& Koelling, R. A. Bait-shyness: A test for toxicity with $\mathrm{N}=2$. Psychonomic Science, 1967, 7, 245.

Garcia, J., McGowan, B. K., Ervin, F. R., \& Koelling, R. A. Cues: Their relative effectiveness as a function of the reinforcer Science, $1868,160,794$

Nachman, M. Learned aversion to the taste of lithium chloride and generalization to other salts. Journal of Comparative \& Phy siological Psychology, 1963, 56, 343-348.

Revusky, S. H., \& Bedarf, E. W. Association of illness with prior ingestion of novel foods. Science, 1967, 155, 210.

(Received for publication February 6, 1973; revision accepted October $4,1973$. 